\title{
Searching gravitational waves from pulsars, using laser beam interferometers
}

\author{
T. Regimbau ${ }^{1}$ and J. A. de Freitas Pacheco ${ }^{2}$ \\ ${ }^{1}$ LIGO laboratory, Massachusetts Institute of Technology, Cambridge, MA 02139, USA \\ 2 Observatoire de la Côte d'Azur, BP 4229, 06304 Nice Cedex 4, France
}

Received 19 August 2002 / Accepted 19 December 2002

\begin{abstract}
We use recent population synthesis results to investigate the distribution of pulsars in the frequency space, having a gravitational strain high enough to be detected by the future generations of laser beam interferometers. We find that until detectors become able to recover the entire population, the frequency distribution of the "detectable" population will be very dependent on the detector noise curve. Assuming a mean equatorial deformation $\varepsilon=10^{-6}$, the optimal frequency is around $450 \mathrm{~Hz}$ for interferometers of the first generation (LIGO or VIRGO) and shifts toward $85 \mathrm{~Hz}$ for advanced detectors. An interesting result for future detection stategies is the significant narrowing of the distribution when improving the sensitivity: with an advanced detector, it is possible to have $90 \%$ of detection probability while exploring less than $20 \%$ of the parameter space $\left(7.5 \%\right.$ in the case of $\left.\varepsilon=10^{-5}\right)$. In addition, we show that in most cases the spindown of 'detectable' pulsars represents a period shift of less than a tens of nanoseconds after one year of observation, making them easier to follow in the frequency space.
\end{abstract}

Key words. gravitational waves - stars: pulsars: general

\section{Introduction}

The coming years will be marked by the beginning of operations for the major gravitational wave interferometric antennas LIGO, VIRGO, GEO and TAMA. Starting with a sensitivity of the order of $h \approx 10^{-22}$, they are expected to evolve in a few years into second generation experiments with a sensitivity improved by one or two orders of magnitude depending on the frequency. Ground based detectors will scan the sky searching for gravitational waves with frequencies between tens of hertz up to tens of kilohertz. Potential sources fall roughly into three classes: bursts, stochastic background, continuous, involving different search techniques as match filtering for coalescing binary systems and cross-correlation between detectors for the stochastic background. The detection of pulsars (and other nearly monochromatic continuous sources) can be achieved by integrating the signal during times of about $10^{7} \mathrm{~s}$ and searching for statistically significant peaks at fixed frequencies in the power spectrum. This method becomes quite complicated as soon as one consider that: 1) pulsars are not really monochromatic emitters. The Doppler effect due to the Earth motion and the intrinsic spindown of the star become significant over long period of times spreading power across many frequency bins and must be corrected before performing the fourier transform. 2) most of pulsars are not observed in the

Send offprint requests to: T. Regimbau, e-mail: tania@ligo.mit.edu electromagnetic domain due to strong selection effects, making necessary an all-sky and all-frequency search. The computational cost is therefore the main concern of searching for continuous sources: if the whole parameter space is scanned, the computing power required to process the data will be out of any reasonable value: of the order of $3 \times 10^{20}$ Tera-flops for integration times of one year, frequency bandwiths of $2500 \mathrm{~Hz}$ and decay times of 1000 year (Frasca et al. 2000). Alternative search algorithms involving hierarchical methods which follow up candidate detections from a first pass search have been investigated during the past few years (Brady et al. 2000; Schutz \& Papa 1999, among others). These techniques reducing very significantly the computing time, by more than ten orders of magnitude for a small loss of sensitivity (by less than an order of magnitude) (Frasca et al. 2000) represent the best hope of detection for actual computational ressources and detector sensitivities. An attempt to optimize these algorithms could be the determination of the most likely range on the parameter space. Without restricting the search area definitively, the probability to have a first detection early could be optimized. Brady et al. (1998) have considered a survey in a limited sky area, searching for pulsars in the galactic core. In a previous work (Regimbau \& de Freitas Pacheco 2000, hereafter Paper I), population synthesis of galactic normal pulsars was performed in order to recover the statistical properties of the real population. This approach permits the modeling of the galactic pulsar contribution to the gravitational emission and to deduce its 
detectability by laser beam interferometers. The gravitational strain being proportional to the star equatorial deformation, the number of detection depends on the mean value adopted for the equatorial ellipticity. This parameter is quite uncertain since it depends on the cooling history and on different strains underwent by the crust. Past studies (Thorne 1980) have shown that equatorial distortions cannot be larger than $10^{-5}$, expected to be an upper limit related to the maximum stress that the solid crust could support, but it is worth mentioning that it could be several orders of magnitude smaller. Our simulations have showed that $10^{-6}$ is the critical value to have at least one detection with interferometers of the first generation $\left(10^{-7}\right.$ for an advanced interferometer) In this paper, by using our previous population synthetis we investigate the detection probability of a pulsar in the frequency space. In order to illustrate our findings, we report here results for mean ellipticities equal to $10^{-5}$ and $10^{-6}$, as well as how the results are affected by the detector sensitivity.

\section{The population synthesis}

\subsection{The model}

Population synthesis aimed to estimate the gravitational contribution of galactic normal pulsars are described in detail in Paper I. The initial parameter distributions being fully taken into account, our statistical approach gives more realistic estimates of the gravitational strain and the number of "potentially detectable" pulsars with frequencies falling into the detector sensitivity band, than other analytical methods. Among the main results of our simulations those relevants for the present purpose are briefly reminded here: a) the average initial period of pulsars is $290 \mathrm{~ms}$ with a dispersion of $100 \mathrm{~ms}$. Pulsars are not born in majority as fast rotators in agreement with recent studies (Bhattacharya et al. 1192; Hartman et al. 1997; Narayan 1987) and with observations, since the mean period of pulsars younger than 20,50 and $100 \mathrm{kyr}$ is respectively 123 , 162 and 173 ms (Lyne \& Granham-Smith 1998). However, the present simulations with a non-fixed initial period permit to account for the fastest objects like the Crab pulsar $(33.4 \mathrm{~ms})$ and for the diversity of young pulsar periods: PSR B1509-58 (as young as the Crab pulsar) has a period of $150 \mathrm{~ms}$, PSR B161050 (six times older) a period of $231 \mathrm{~ms}$, while the VELA pulsar and PSR B1951+32, respectively 10 and 100 times older than the Crab pulsar rotate with periods of $89 \mathrm{~ms}$ and $59 \mathrm{~ms}$. b) around 150000 pulsars are expected to be present in the Galaxy. c) among them, pulsars in the detector frequency range of sensitivity (called later "potentially detectables") would be around 1000 if the lower bound is $10 \mathrm{~Hz}$ or around 5200 if it is $5 \mathrm{~Hz}$. The latter is expected to be attained in a second phase of the VIRGO experiment, thanks to a sophisticated pendulum system called superatenuator. Notice that the upper bound of the detector (around 5-10 kHz) does not interpose on the detection of pulsars, since the Keplerian stability limit of neutron stars corresponds to gravitational wave frequencies of $2-4 \mathrm{kHz}$, depending on the equation of state.

\subsection{The detectable population}

When any precessional effect is neglected the two polarization components of gravitational waves emitted by a rotating neutron star are (Bonazzola \& Gourgoulhon 1996)

$h_{+}(t)=2 A\left(1+\cos ^{2} i\right) \cos (2 \Omega t)$

and

$h_{\times}(t)=4 A \cos i \sin (2 \Omega t)$

where $i$ is the angle between the spin axis and the wave propagation vector, assumed to coincide with the line of sight, and the wave amplitude is defined by

$A=\frac{G}{r c^{4}} \varepsilon I_{z z} \Omega^{2}$.

In this expression, $G$ is the gravitation constant, $c$ is the velocity of light, $r$ is the distance to the source, $\Omega$ is the angular rotation velocity of the pulsar and the ellipticity $\varepsilon$ is defined as

$\varepsilon=\frac{I_{x x}-I_{y y}}{I_{z z}}$.

The gravitational strain amplitude induced in the detector depends also on the direction and polarization of the wave with relative to the detector arms and it is given by:

$h(t)=h_{+}(t) F_{+}(\theta, \phi, \psi)+h_{\times}(t) F_{\times}(\theta, \phi, \psi)$

where $F_{+, \times}$are the beam factors of the interferometer (Jaranowski et al. 1998), which are functions of the zenith distance $\theta$, the azimuth $\phi$ as well as of the wave polarization plane orientation $\psi$.

Among the "potentially detectable" population mentioned above (pulsars with periods less than $0.4 \mathrm{~s}$ for VIRGO and less than $0.2 \mathrm{~s}$ for LIGO), "detectable" objects are pulsars with gravitational strain amplitudes above the antenna sensitivity curve. The detectability criterium can be expressed as follows:

$h\left(f_{g}\right) \geq 2 \frac{h_{n}\left(f_{g}\right)}{\sqrt{T}}$

where $h$ is the gravitational strain, $T$ the integration time, $h_{n}$ the detector sensitivity (in $\mathrm{Hz}^{-1 / 2}$ ). In our analysis, the planned LIGO II was used as an exemple of second generation detectors. The factor two corresponds to the adopted signal-to-noise ratio. As one should expect, the less is $\varepsilon$, the harder is the detectability condition. The number of detections as a function of the ellipticity and detector sensitivity is reported in Table 1 . An average ellipticity of $10^{-6}$ represents the detectability threshold for detectors of the first generation as VIRGO or LIGO I: around $12-15$ detections are expected if $\varepsilon=10^{-5}, 2-3$ detections if $\varepsilon=10^{-6}$ and no detection if $\varepsilon<10^{-6}$. For an advanced interferometer this threshold corresponds to a mean ellipticity of $10^{-7}$. This result has quite interesting consequences: even in the case of no detection, an upper limit can be set to the ellipticity and improvements in the sensibility will gradually push down such a limit.

As shown in Table 1, detectable pulsars are expected to be few at the beginning, since only the nearest and the fastest objects have strain amplitudes high enough to be above the noise 
Table 1. Number of detections as a function of the ellipticity for interferometers of the first generation (LIGO and VIRGO) and for an advanced interferometer.

\begin{tabular}{lccc}
\hline \hline$\varepsilon$ & $10^{-5}$ & $10^{-6}$ & $10^{-7}$ \\
\hline VIRGO & 15 & 3 & 0 \\
LIGO I & 12 & 2 & 0 \\
Advanced & 90 & 12 & 2 \\
\hline
\end{tabular}

level. The detector sensitivity acts as a filter affecting a priori both spatial and frequency statistics. Actually our simulations have shown that the distance distribution is quite similar for the detectable population and the total population, with a peak around the galactic center. As a result the sky direction distributions do not depend significantly on the antenna sensitivity or on the ellipticity. On the opposite, the frequency distribution is very dependent on both of them and requires a more detailed analysis.

\subsection{The gravitationnal frequency}

The gravitational frequency distribution of detectable pulsars as a function of the antenna sensitivity, for two different values of the ellipticity, the theoretical upper value $\varepsilon=10^{-5}$ and the detectability limit for interferometers of the first generation, $\varepsilon=10^{-6}$ are shown respectively in Figs. 1 and 2 .

The sensitivity improvement enables the detection of slower objects, so the maximum of the detectable frequency distribution shifts toward lower values. For $\varepsilon=10^{-5}$, the maximum is respectively around $180 \mathrm{~Hz}$ and $135 \mathrm{~Hz}$ for VIRGO and LIGO I and decreases to $85 \mathrm{~Hz}$ for an advanced interferometer. For $\varepsilon=10^{-6}$ it is $480 \mathrm{~Hz}$ for VIRGO, $435 \mathrm{~Hz}$ for LIGO I and $85 \mathrm{~Hz}$ for for an advanced interferometer. The difference between the optimal frequencies of the two detectors of the first generation arises from the fact that LIGO I is more sensitive than VIRGO between $50 \mathrm{~Hz}$ and $330 \mathrm{~Hz}$ and less sensitive elsewhere. What is interesting to notice for future detection stategy, is the significant reduction of the distribution width between the first and second generation (see Table 2). It decreases from $200 \mathrm{~Hz}$ to $50 \mathrm{~Hz}$ if $\varepsilon=10^{-5}$ and from $700-1000 \mathrm{~Hz}$ to $200 \mathrm{~Hz}$ if $\varepsilon=10^{-6}$. Consequently, we could keep $90 \%$ of the maximum detection probability while exploring less than $7.5 \%$ of the frequency space with an advanced detector versus $25 \%$ with interferometers of the first generation. Another important result concerns the lower bound of the detector frequency band. At the begining, this value does not play much part but the improvement of the sensitivity would step by step allow one to recover the entire "potentially detectable" population concentrated at the lowest frequencies.

As already mentioned, one of the major complications when searching for pulsars over long periods of time arises from the intrinsic spindown which spreads power across many frequency bins. A model of the intrinsic frequency evolution is thus needed to remove these effects before performing the Fourier transform. Estimates of the spindown parameters can be derived from observations of radio pulsars

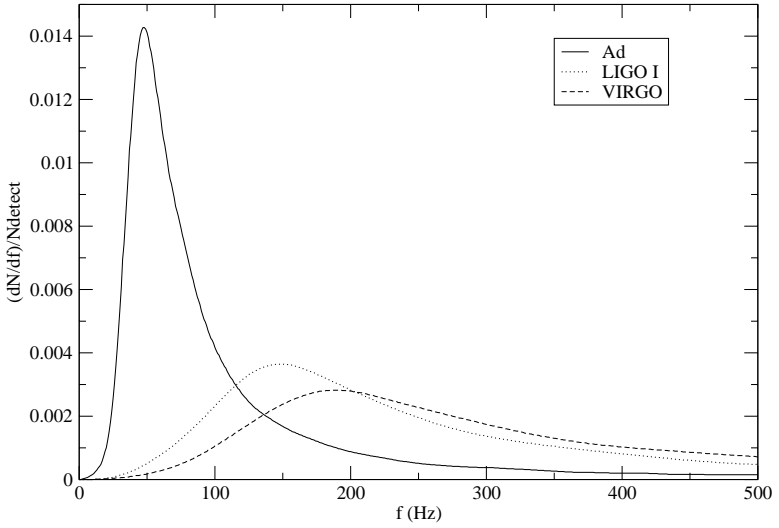

Fig. 1. Frequency distribution of detectable pulsars if $\varepsilon=10^{-5}$.

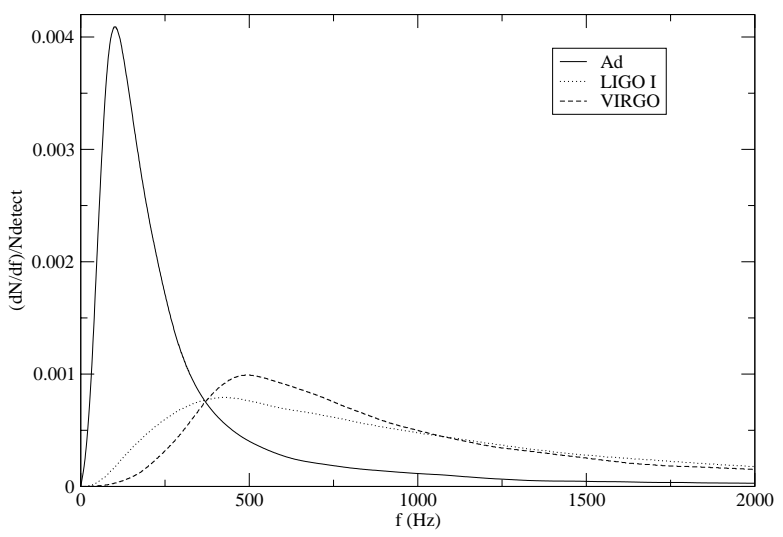

Fig. 2. Frequency distribution of detectable pulsars if $\varepsilon=10^{-6}$.

(Brady et al. 1998 and 2000 for instance) but strong selection effects make them non-representative of the detectable population. In the following, we investigate the statistical properties of the spindown rate of detectable pulsars. The period derivative distribution of detectable pulsars as a function of the antenna sensitivity and for an ellipticity $\varepsilon=10^{-6}$ is shown in Fig. 3 and compared with the expected distribution of the "potentially detectable" population. As there is no significant difference between the distributions of the first generation interferometers, only one plot is reported for both LIGO I and VIRGO.

The period derivative probability function can be represented quite well by a Gaussian distribution with standard deviation $\sigma=0.6$. The maximum (or the mean value) is around $\log (\dot{P})=-17.5$ for interferometers of the first generation and increases to $\log (\dot{P})=-16.8$ for an advanced interferometer. As the sensitivity improves, the distribution shifts toward the expected distribution of the "potentially detectable" population which maximum is $\log (\dot{P})=-15.6$. Changing the mean ellipticity from $\varepsilon=10^{-6}$ to $\varepsilon=10^{-5}$ acts the same way (see Table 3 ) The general belief is that detectable pulsars will be the youngest at the beginning of the operations but it is shown here that it is not necessarily the case. Actually, detectable pulsars are usually the "most stable". That is very understandable considering that the less its spindown, the longer a pulsar remains in the high frequency range, which increase its probability of being detected. 
Table 2. Parameters of the detectable frequency distribution for $\varepsilon=$ $10^{-5}$ (left value) and $\varepsilon=10^{-6}$ (right value) for interferometers of the first generation (LIGO and VIRGO) and for an advanced interferometer: the maximum, the width and the frequency band around the maximum where we find $90 \%$ of detection.

\begin{tabular}{lccc}
\hline \hline & $\max (\mathrm{Hz})$ & width $(\mathrm{Hz})$ & $90 \%$ band $(\mathrm{Hz})$ \\
\hline VIRGO & $180-480$ & $200-700$ & {$[0-1200]-[0-2500]$} \\
LIGO I & $135-435$ & $200-1000$ & {$[0-1000]-[0-2600]$} \\
Advanced & $45-85$ & $50-200$ & {$[0-300]-[0-2500]$} \\
\hline
\end{tabular}

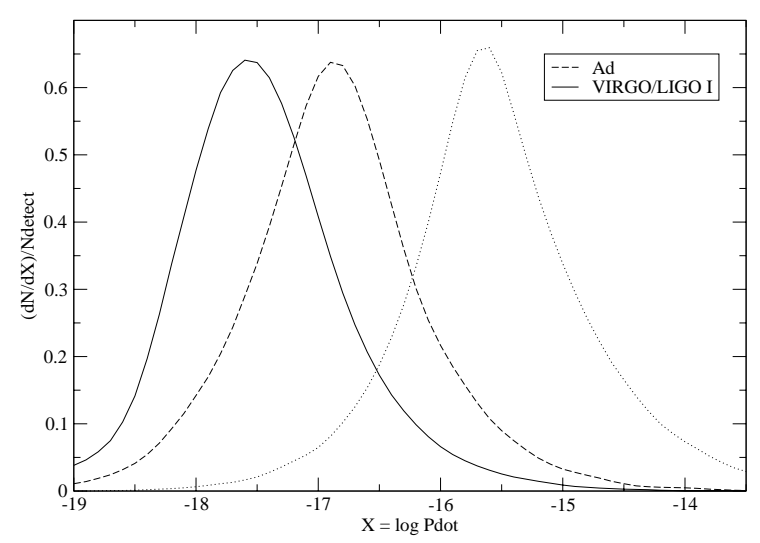

Fig. 3. Period derivative distribution of the detectable pulsar population if $\varepsilon=10^{-6}$ for the sensitivity of interferometers of the first generation (plain line) as well as for an advanced interferometer (dashed line). The dot line represents the expected distribution of the "potentially" detectable population.

It is relevant to define the critical spindown rate $\dot{P}_{90 \%}$ below which $90 \%$ of detectable pulsars can be found. This parameter may be used to determine the number of bins that should be investigated in the frequency space to correct spindown effects. Table 3 gives $\dot{P}_{90 \%}$ as well as a more conservative $\dot{P}_{99 \%}$, for interferometers of the first and second generations and for ellipticities $\varepsilon=10^{-5}$ and $10^{-6}$. Columns $\Delta P_{90 \%}$ and $\Delta P_{99 \%}$ give an estimation of the maximal shift one should expect (at $90 \%$ and $99 \%$ of confidence) after one year of observation (Eq. (7) with $T=1 \mathrm{yr}$ )

$\Delta P_{90 \%}=\dot{P}_{90 \%} \times T$.

\subsection{Conclusions}

In this paper, the distribution in the frequency space of pulsars with a gravitational strain high enough to be detected by the future generations of laser beam interferometers was investigated. Until detectors become able to recover the entire population, the "detectable" frequency distribution will be dependent
Table 3. Parameters of the period derivative distribution for $\varepsilon=10^{-6}$ (left value) and $\varepsilon=10^{-5}$ (in parenthesis) for interferometers of the first generation and for an advanced interferometer: the maximum, the 0.9 and 0.99 quantiles (lines 3 and 5) and the corresponding period shifts in nanoseconds after one year of observation (lines 4 and 6).

\begin{tabular}{lcc}
\hline \hline & LIGO/VIRGO & Advanced \\
\hline $\max$ & $-17.5(-17.2)$ & $-16.8(-16.4)$ \\
$\log \dot{P}_{90 \%}$ & $-16.7(-16.4)$ & $-16.0(-15.6)$ \\
$\Delta P_{90 \%}(\mathrm{~ns})$ & $0.63(1.26)$ & $3.15(7.93)$ \\
$\log \dot{P}_{99 \%}$ & $-16.1(-15.8)$ & $-15.4(-15)$ \\
$\Delta P_{99 \%}(\mathrm{~ns})$ & $2.51(5.00)$ & $12.65(31.56)$ \\
\hline
\end{tabular}

on the detector sensitivity. If $\varepsilon=10^{-6}$, the optimal frequency is around $450 \mathrm{~Hz}$ for interferometers of the first generation and shifts toward $75 \mathrm{~Hz}$ for an advanced detector. An encouraging result for future detection stategies is the significant narrowing of the optimal band of frequency which follows the improvement of the sensitivity: with an advanced detector we could keep $90 \%$ of the maximum detection probability while exploring less than $20 \%$ of the frequency space $\left(7.5 \% \varepsilon=10^{-5}\right)$. In addition, we showed that in most cases "detectable" pulsars have very stable rotations, making them easier to track in the frequency space. The critical spindown rate below which $90 \%$ of detectable pulsars can be found is of the order of a few nanoseconds per year for interferometers of the first generation (and tens of nanoseconds per year for an advanced interferometer). This informations integrated into detection algorithms may be useful to choose the optimal way to start blind searches, increasing our chances of detecting these objects.

\section{References}

Bhattacharya, D., Wijers, R., Hartman, J. W., \& Verbunt, F. 1992, A\&A, 254, 198

Bonazzola, S., \& Gourgoulhon, E. 1996, A\&A, 312, 675

Brady, P. R., Creighton, T., Cutler, C., \& Schutz, B. F. 1998, Phys. Rev. D, 57,4

Brady, P. R., \& Creighton, T. 2000, Phys. Rev. D, 61, 8

Frasca, S. 2000, Int. J. Mod. Phys. D, 9, 369

Hartman, J. W., Bhattacharya, D., Wijers, R., \& Verbunt, F. 1997, A\&A, 322, 477

Jaranowski, P., Królak, A., \& Schutz, B. F. 1998 [gr-qc/9804014]

Lyne, A. G., \& Graham-Smith, F. 1998, in Pulsar Astronomy (Cambridge: Cambridge University Press)

Narayan, R. 1987, ApJ, 319, 162

Regimbau, T., \& de Freitas Pacheco, J. A. 2000, A\&A, 359, 242, Paper I

Schutz, B. F., \& Papa, M. A. 1999 [gr-qc/9905018]

Thorne, K. S. 1980, Rev. Mod. Phys., 52, 299

Zimmerman, M., \& Szedenits, E. 1979, Phys. Rev. D, 20, 351 